ARQGA / 954

\title{
ALTERAÇÕES NO TESTE ULTRA-RÁPIDO DA UREASE E NO EXAME ANATOMOPATOLÓGICO PARA Helicobacter pylori INDUZIDAS POR DROGAS ANTI-SECRETORAS
}

\author{
Lincoln Eduardo Villela Vieira de Castro FERREIRA ${ }^{1}$, Gustavo de Souza Portes MEIRELLES ${ }^{2}$, \\ Renata La Rocca VIEIRA ${ }^{3}$, Maurício Augusto BRAGAGNOLO Jr. ${ }^{4}$, \\ Júlio Maria Fonseca CHEBLI ${ }^{5}$ e Aécio Flávio Meirelles de SOUZA ${ }^{6}$
}

RESUMO - Racional - Um dos problemas na avaliação de pacientes dispépticos em instituições públicas é o intervalo de tempo prolongado entre a consulta inicial e a endoscopia digestiva alta, levando à introdução de terapêutica anti-secretora nesse período. Desta forma, os resultados do exame podem não traduzir a condição inicialmente suspeitada. Objetivos Analisar as alterações no teste ultra-rápido da urease e no exame anatomopatológico para Helicobacter pylori após o uso de drogas anti-secretoras. Casuística e Método - Foram avaliados, de forma prospectiva e duplo-cega, 50 pacientes com queixas dispépticas e diagnóstico endoscópico de úlcera péptica, gastrite, esofagite ou duodenite erosivas e com teste da urease positivo. Os pacientes foram divididos, de forma randomizada, em dois grupos: 25 usaram ranitidina $300 \mathrm{mg} /$ dia e 25, omeprazol $20 \mathrm{mg} /$ dia, durante 7 dias. Antes e após o tratamento, dois fragmentos de biopsia foram obtidos do antro e corpo gástricos, realizando-se o teste ultra-rápido da urease e o exame anatomopatológico para Helicobacter pylori. Resultados - No grupo que usou ranitidina, não se observaram alterações significativas na positividade do teste ultrarápido da urease e do exame anatomopatológico no antro e corpo gástricos, após 7 dias de medicação. Nos pacientes que utilizaram omeprazol, após tal período, observou-se redução da positividade do teste ultra-rápido da urease e do exame anatomopatológico no antro gástrico, sendo que o mesmo não ocorreu na região do corpo. Conclusão - O omeprazol, utilizado por um período de 7 dias, é capaz de levar a uma negativação do teste ultra-rápido da urease e do exame anatomopatológico para Helicobacter pylori no antro gástrico, devendo ser desaconselhado para pacientes aguardando realização de endoscopia digestiva,

DESCRITORES - Antiulcerosos. Infecções por helicobacter. Omeprazol. Ranitidina. Helicobacter pylori.

\footnotetext{
Serviço de Gastroenterologia do Hospital Universitário da Universidade Federal de Juiz de Fora - UFJF, Juiz de Fora, MG. Médico do Setor de Endoscopia Digestiva do Serviço de Gastroenterologia da UFJF. Médico Residente da Universidade Federal de São Paulo - Escola Paulista de Medicina. Acadêmica de Medicina da UFJF. Bolsista de iniciação científica do CNPq. Médico Residente de Gastroenterologia Clínica do Hospital Universitário da UFJF.

Professor Adjunto da Disciplina de Gastroenterologia da Faculdade de Medicina da UFJF.

Professor Adjunto da Disciplina de Gastroenterologia da Faculdade de Medicina da UFJF. Chefe do Serviço de Gastroenterologia do Hospital Universitário da UFJF e do Núcleo de Estudos e Pesquisas em Gastroenterologia e Endoscopia Digestiva.

Endereço para correspondência: Dr. Gustavo de Souza Portes Meirelles. Rua Doutor Diogo de Faria, 650/33 - Vila Clementino - 04037-002 - São Paulo, SP. e-mail: gm@rural.com.br
} 


\section{INTRODUÇÃO}

O primeiro relato conhecido da presença de bactérias espiraladas no estômago de animais deve-se a Krienitz, em 1906. Entretanto, nessa época, acreditava-se que estas fossem apenas comensais. Somente após os trabalhos de WARREN e MARSHALL ${ }^{(10,11,27)}$, em 1983, chamando a atenção para a freqüente associação entre o Helicobacter pylori (H.pylori), gastrite crônica e úlcera péptica, é que foi postulada a participação do microrganismo na gênese destas afecções.

O diagnóstico do H. pylori se faz através de métodos microbiológicos, histológicos, sorológicos, bioquímicos e isotópicos ${ }^{(7,12,19)}$. Dentre os métodos utilizados, alguns se baseiam na detecção da urease, enzima produzida em grande quantidade pela bactéria. A técnica consiste na coleta de fragmentos de mucosa do antro gástrico de pacientes submetidos a endoscopia digestiva alta e colocação destes num meio que contenha uréia e um indicador de $\mathrm{pH}$. Quando o H. pylori está presente na mucosa antral colhida, a urease por ele produzida desdobra a uréia do meio, produzindo amônia, levando a aumento do $\mathrm{pH}$ e a mudança na cor do indicador ${ }^{(15)}$. Alguns testes levam em consideração os resultados até 2 horas após a colocação do fragmento no meio, enquanto o teste ultra-rápido o faz em apenas 1 minuto $^{(1,7,15,26)}$. Este tem sensibilidade de $89 \%$ a $91 \%$, especificidade de $100 \%$, com valor preditivo positivo de $94 \%$ e negativo de $100 \%$, com acurácia de $96 \%{ }^{(7,15,26)}$, comparável aos demais testes utilizados na detecção do H. pylori, como a cultura, histopatologia, testes sorológicos, teste da urease convencional e teste respiratório com uréia marcada por Carbono ${ }^{14}$, que têm sensibilidade e especificidade variando de $90 \%$ a $98 \%{ }^{(15,19)}$. A grande vantagem do teste ultra-rápido da urease é a rapidez de seu resultado, uma vez que a leitura é feita com apenas 1 minuto, e seu baixíssimo custo $^{(1,7,26)}$. Os demais testes necessitam de maior tempo para obter o resultado. A cultura, por exemplo, requer de 3 a 6 dias para o isolamento do microorganismo.

Tanto o omeprazol quanto o lansoprazol e o pantoprazol (e seus metabólitos ativos) apresentam atividade antimicrobiana in vitro contra o H. pylori, por inibirem a urease da bactéria. A elevação do pH após a administração de inibidor da bomba protônica, aumenta a concentração e prolonga o efeito dos antibióticos ácido-lábeis. $\mathrm{O}$ pH elevado também aumenta os mecanismos de defesa do hospedeiro, incluindo aumento da concentração e meia-vida das imunoglobulinas específicas para o H. pylori no suco gástrico, melhorando a função dos neutrófilos, necessária para se conseguir a erradicação do microrganismo ${ }^{(6,18,22,24,25)}$.

Administrado isoladamente, o omeprazol suprime temporariamente o H. pylori do antro ${ }^{(3,8,28)}$, o que concorre para taxas de recurrência superiores àquelas dos pacientes tratados com a associação do inibidor de bomba protônica e antimicrobianos ${ }^{(4)}$. Com o uso prolongado do omeprazol, instituído no tratamento de afecções como esofagite erosiva, a densidade de bactérias diminui no antro e aumenta na mucosa do corpo gástrico, o que pode ser fator desencadeante de gastrite atrófica ${ }^{(23)}$. Tal fato tem levado alguns autores a preconizarem a erradicação do $H$. pylori nesses pacientes ${ }^{(2,3,8,23)}$. Sabe-se ainda que, em portadores de úlcera duodenal portadores do H. pylori, a utilização do omeprazol por 2 semanas pode cicatrizar a lesão em $40 \%$ a $80 \%$ das vezes, além de levar ao desaparecimento do H. pylori em até $50 \%$ dos $\operatorname{casos}^{(5,14,17)}$. Portanto, se o exame endoscópico for realizado após este prazo, o laudo endoscópico e o teste rápido da urease poderão não traduzir a condição clínica inicialmente suspeitada pelo médico assistente.

Com relação aos antagonistas dos receptores $\mathrm{H}_{2}$, como a ranitidina, acredita-se que não apresentem atividade antimicrobiana direta sobre o H. pylori. Entretanto, após seu uso prolongado, são capazes de levar a um aumento da extensão da gastrite na região do corpo gástrico, o que levou alguns autores a sugerirem a pesquisa do $H$. pylori antes do uso prolongado com tais drogas ${ }^{(16)}$.

Os objetivos deste estudo foram correlacionar as alterações no teste ultra-rápido da urease e na pesquisa anatomopatológica para $H$. pylori, antes e após o tratamento com drogas anti-secretoras.

\section{CASUÍSTICA E MÉTODO}

O estudo foi realizado junto ao setor de Endoscopia Digestiva do Serviço de Gastroenterologia do Hospital Universitário da Universidade Federal de Juiz de Fora (UFJF), no período de agosto de 1998 a julho de 1999, do qual fizeram parte pacientes encaminhados pelo ambulatório geral de Gastroenterologia, com queixas de dispepsia (dor epigástrica em queimação ou tipo "vazio", náuseas, vômitos, pirose e plenitude pós-prandial), com diagnóstico endoscópico de úlcera péptica e/ou gastrite erosiva e/ou duodenite erosiva e/ou esofagite erosiva, independente do grau das lesões, com teste da urease positivo para $H$. pylori. Foram excluídos do trabalho os pacientes: em uso atual ou recente (últimos 90 dias) de antimicrobianos com atividade contra o H. pylori, bloqueadores de bomba protônica, antagonistas dos receptores $\mathrm{H}_{2}$ ou subcitrato de bismuto coloidal; tratados para H. pylori nos últimos 6 meses; portadores de hepatopatia crônica e/ou hipertensão portal; submetidos a gastrectomia total ou parcial e aqueles com história de cirurgia para o tratamento da úlcera péptica. De acordo com esses critérios, 50 pacientes foram incluídos na pesquisa. A média de idade do grupo estudado foi de $47,6 \pm 14$ anos, variando de 19 a 76 anos. Trinta $(60,0 \%)$ dos pacientes pertenciam ao sexo feminino e $20(40,0 \%)$ ao sexo masculino. Trinta e quatro $(68,0 \%)$ dos pacientes eram de cor branca.

Todos foram submetidos a endoscopia digestiva alta (Olympus EVIS 100), permanecendo sem medicação anti-secretora no intervalo 
entre a consulta inicial e o exame endoscópico. Durante a endoscopia digestiva alta (EDA), cujo diagnóstico era devidamente anotado, foram retirados dois fragmentos da mucosa antral e dois da mucosa de corpo para a realização do teste ultra-rápido da urease, além de um fragmento de antro e outro de corpo para a pesquisa histológica do H. pylori. O teste ultra-rápido da urease foi realizado baseado no método descrito por ARVIND et al. ${ }^{(1)}$. Após a biopsia, os fragmentos eram colocados imediatamente num tubo contendo $1 \mathrm{~mL}$ de água destilada e uréia a $10 \%$, com $\mathrm{pH}$ em torno de 6,8 , utilizando-se como indicador de $\mathrm{pH}$ duas gotas de vermelho fenol a $1 \%$. Considerou-se como resultado positivo a mudança de cor da solução de amarelo para rosa, dentro de 1 minuto. Para a análise histológica, empregou-se a coloração de Giemsa $^{(12,19)}$.

Após explicação detalhada do projeto, com exposição dos riscos e benefícios de um novo exame endoscópico ${ }^{(13,20)}$ e da medicação a ser empregada, os pacientes que concordaram em participar do estudo assinaram um termo de consentimento. Foram divididos em dois grupos: de forma randomizada e duplo-cega; o primeiro recebeu um antagonista do receptor $\mathrm{H}_{2}$ (ranitidina, $300 \mathrm{mg} / \mathrm{dia}$ ); o segundo, um inibidor da bomba de prótons (omeprazol, $20 \mathrm{mg} / \mathrm{dia}$ ), ambos administrados pela manhã, em jejum.

Os pacientes e os médicos envolvidos na avaliação endoscópica e anatomopatológica não tiveram conhecimento da droga que estava sendo utilizada. Os pacientes retornaram após 7 dias da primeira EDA para novo exame endoscópico e novo teste da urease. Durante a segunda EDA foram retirados novamente dois fragmentos da mucosa antral e dois da mucosa de corpo para a realização do teste ultrarápido da urease, e um fragmento de antro e outro de corpo para a pesquisa histológica do H. pylori. A leitura do teste ultra-rápido da urease foi novamente feita após 1 minuto.

Compararam-se os testes da urease e os estudos anatomopatológicos nos dois momentos (inicial e após 7 dias de tratamento). Os pacientes foram, então, encaminhados para acompanhamento ambulatorial, com posterior avaliação clínica e instituição de terapêutica adequada.

Para a análise estatística, por se tratarem de amostras pareadas não-independentes, utilizou-se o teste de $\mathrm{McNemar}^{(21)}$, adotando-se um intervalo de confiança de $95 \%(P<0,05)$.
O protocolo de estudo foi submetido a análise pela Comissão de Ética Médica do Hospital Universitário da UFJF, sendo aprovado sem objeções.

\section{RESULTADOS}

O achado endoscópico mais freqüente foi a gastrite erosiva (65,3\%), seguida por esofagite erosiva $(34,5 \%)$, duodenite erosiva $(29,5 \%)$, úlcera duodenal (16,6\%) e úlcera gástrica (12,3\%). Alterações inflamatórias mínimas (gastrite e duodenite não-erosivas) foram encontradas em 18,8\% dos casos. Alguns pacientes apresentaram mais de uma lesão ao exame endoscópico; por esta razão, seu número de achados foi superior ao número total de pacientes.

Dos 50 pacientes estudados, 25 fizeram uso de ranitidina e os demais, de omeprazol. Todos aceitaram bem a medicação, sendo que a quase totalidade $(93 \%)$ relatou melhora clínica.

Grupo 1 - Pacientes que usaram ranitidina - Fizeram parte deste grupo 25 pacientes, sendo $16(64 \%)$ do sexo feminino e $9(36 \%)$, do sexo masculino. A média de idade foi de 49,8 $\pm 10,1$, variando de 31 a 66 anos. Dezesseis (64\%) pacientes eram de cor branca.

Na primeira endoscopia a positividade do teste ultra-rápido da urease foi de $88 \%$ ( 22 pacientes) no antro e $68 \%$ (17 pacientes) no corpo. Com relação ao exame anatomopatológico, a pesquisa do $H$. pylori foi positiva em 24 pacientes (96\%) no antro e 20 (80\%) no corpo.

Após 7 dias de uso da ranitidina, repetiu-se o exame endoscópico, observando-se positividade do teste ultra-rápido da urease de $76 \%$ (19 pacientes) no antro e 56\% (14 pacientes) no corpo. A pesquisa anatomopatológica do $H$. pylori foi positiva em 22 pacientes $(88 \%)$ no antro e 23 pacientes ( $92 \%$ ) no corpo.

Comparando-se os dois exames endoscópicos (Tabelas 1,2), verificou-se não haver diferença significativa, do ponto de vista estatístico, entre a positividade do teste ultra-rápido da urease, bem como da pesquisa anatomopatológica para H. pylori, no antro e corpo, antes e após o uso de ranitidina por 7 dias $(P>0,05)$.

Grupo 2 - Pacientes que usaram omeprazol - Constaram deste grupo 25 pacientes, sendo 14 (56\%) do sexo feminino e 11 (44\%) do sexo masculino. A média de idade foi de $45,4 \pm 16,9$, variando de 19 a 76 anos. Dezoito pacientes (72\%) eram de cor branca.

TABELA 1 - Positividade do teste ultra-rápido da urease antes e após o uso de ranitidina, no antro e corpo gástricos

\begin{tabular}{ccccc}
\hline & $\mathbf{1}^{\mathbf{a}}$ EDA & $\mathbf{2}^{\mathbf{a}} \mathbf{E D A}$ & $\boldsymbol{\chi}^{2}$ & $\boldsymbol{P}$ \\
\hline \multirow{3}{*}{ Antro } & $\mathrm{N}^{\mathrm{o}}(\%)$ & $\mathrm{N}^{\mathrm{o}}(\%)$ & & $>0,05$ \\
\cline { 2 - 5 } Corpo & $22(88,0)$ & $19(76,0)$ & 1,22 & $>0,05$ \\
\hline
\end{tabular}


TABELA 2 - Positividade da pesquisa anatomopatológica antes e após o uso de ranitidina, no antro e corpo gástricos

\begin{tabular}{ccccr}
\hline & $\mathbf{1}^{\mathbf{a}} \mathbf{E D A}$ & $\mathbf{2}^{\mathbf{a}} \mathbf{E D A}$ & $\boldsymbol{\chi}^{2}$ & \multicolumn{1}{c}{} \\
\cline { 2 - 5 } & $\mathbf{N}^{\mathbf{0}} \mathbf{( \% )}$ & $\mathbf{N}^{\mathbf{0}} \mathbf{( \% )}$ & & $>0,05$ \\
\cline { 2 - 5 } Antro & $24(96,0)$ & $22(88,0)$ & 1,09 & $>0,05$ \\
Corpo & $20(80,0)$ & $23(92,0)$ & 1,50 & \\
\hline
\end{tabular}

A positividade do teste ultra-rápido da urease foi de $100 \%$ (25 pacientes) no antro e $64 \%$ ( 16 pacientes) no corpo, na primeira endoscopia. Com relação ao exame anatomopatológico, a pesquisa do $H$. pylori foi positiva em 23 pacientes (92\%) no antro e 21 (84\%) no corpo.

Realizou-se novo exame endoscópico, após 7 dias de uso do omeprazol, observando-se uma positividade do teste ultra-rápido da urease de $64 \%$ (16 pacientes) no antro e 76\% (19 pacientes) no corpo.
A pesquisa anatomopatológica do H. pylori foi positiva em 10 pacientes (40\%) no antro e em 14 pacientes (56\%) no corpo.

Analisando-se o teste ultra-rápido da urease (Tabela 3) e o exame anatomopatológico para H. pylori (Tabela 4), verificou-se haver redução estatisticamente significativa na positividade de ambos no antro gástrico $(P<0,001)$, após o uso de omeprazol por 7 dias, sendo que o mesmo não foi observado no corpo $(P>0,05)$.

TABELA 3 - Positividade do teste ultra-rápido da urease antes e após o uso de omeprazol, no antro e corpo gástricos

\begin{tabular}{ccccc}
\hline & $\mathbf{1}^{\mathbf{a}} \mathbf{E D A}$ & $\mathbf{2}^{\mathbf{a}} \mathbf{E D A}$ & $\boldsymbol{\chi}^{\mathbf{2}}$ & \multicolumn{1}{c}{} \\
\cline { 2 - 5 } & $\mathbf{N}^{\mathbf{0}} \mathbf{( \% )}$ & $\mathbf{N}^{\mathbf{0}} \mathbf{( \% )}$ & & $<0,001$ \\
\cline { 2 - 5 } Antro & $25(100,0)$ & $16(64,0)$ & 10,98 & $>0,05$ \\
Corpo & $16(64,0)$ & $19(76,0)$ & 0,86 & $>06$ \\
\hline
\end{tabular}

TABELA 4 - Positividade da pesquisa anatomopatológica antes e após o uso de omeprazol, no antro e corpo gástricos

\begin{tabular}{ccccc}
\hline & $\mathbf{1}^{\mathbf{a}} \mathbf{E D A}$ & $\mathbf{2}^{\mathbf{a}} \mathbf{E D A}$ & $\boldsymbol{\chi}^{\mathbf{2}}$ & \multicolumn{1}{c}{} \\
\cline { 2 - 5 } & $\mathbf{N}^{\mathbf{0}} \mathbf{( \% )}$ & $\mathbf{N}^{\mathbf{0}}(\mathbf{\%})$ & & $<0,001$ \\
\cline { 2 - 5 } Antro & $23(92,0)$ & $10(40,0)$ & 15,06 & $>0,05$ \\
Corpo & $21(84,0)$ & $14(56,0)$ & 3,43 & $>$ \\
\hline
\end{tabular}

\section{DISCUSSÃO}

A maioria dos pacientes com queixas dispépticas atendidos pelo Sistema Único de Saúde (SUS) é submetida a EDA com longo intervalo de tempo após a consulta inicial. Em muitos casos, opta-se pelo início da medicação anti-secretora antes da realização do exame, de modo a reduzir sua sintomatologia clínica enquanto aguardam pela realização do exame. Entretanto, o emprego de tais drogas antes do exame endoscópico pode ocasionar alterações não só no diagnóstico endoscópico do paciente, mas também no teste ultra-rápido da urease e na pesquisa anatomopatológica do $H$. pylori, fazendo com que a condição inicialmente suspeitada pelo médico possa não ser traduzida no momento do exame.

A segunda EDA, realizada após uma semana, não é procedimento de rotina no nosso meio, pois resulta em aumento dos custos e em sobrecarga dos serviços de endoscopia digestiva ${ }^{(9)}$. Entretanto, constitui método valioso no acompanhamento clínico e no controle de cura dos pacientes, não acarretando em agravos à saúde dos mesmos ou em aumento da morbidade e da mortalidade ${ }^{(12,17)}$.
Utilizando drogas de uso freqüente na prática médica, verificou-se negativação do teste ultra-rápido da urease e da pesquisa anatomopatológica do H. pylori no antro gástrico, com o uso de omeprazol $20 \mathrm{mg} /$ dia por 7 dias. Tal alteração parece ser indicativa de supressão temporária local da bactéria, decorrente de provável efeito bactericida do omeprazol ${ }^{(3,10)}$. Essas alterações não foram observadas na região do corpo, talvez por ocorrerem somente com o emprego mais prolongado desta droga ${ }^{(2,3,8)}$.

Com relação à ranitidina — $300 \mathrm{mg} / \mathrm{dia}$ — não foram observadas alterações no teste ultra-rápido da urease e na pesquisa anatomopatológica do H. pylori tanto no antro, quanto no corpo gástrico, após terapêutica por 7 dias. Contudo, não se pode descartar tais alterações no caso de doses maiores ou tempo de tratamento mais prolongado, conforme demonstrado em outros trabalhos ${ }^{(16)}$.

\section{CONCLUSÃO}

O omeprazol, utilizado por um período de 7 dias, é capaz de levar à negativação do teste ultra-rápido da urease e do exame anatomopatológico para H. pylori no antro gástrico, devendo ser droga 
desaconselhada para pacientes aguardando realização de EDA, pelo risco de falsear os resultados dos exames. A ranitidina não levou a alterações no teste ultra-rápido ou no exame anatomopatológico para H. pylori tanto em antro, quanto em corpo gástrico, quando utilizada pelo mesmo período de tempo.

\section{AGRADECIMENTOS}

Os autores agradecem ao Conselho Nacional de Desenvolvimento Científico e Tecnológico (CNPq) e à Fundação de Amparo à Pesquisa do Estado de Minas Gerais (FAPEMIG).

Ferreira LEVV de C, Meirelles G de SP, Vieira R La R, Bragagnolo Jr. MA, Chebli JMF, Souza AFM de. Changes in ultrarapid urease test and histopathological examination for Helicobacter pylori by antisecretory drugs. Arq Gastroenterol 2001;38(1):3-8.

ABSTRACT - Background - One of the major problems when evaluating dyspeptic patients at public hospitals is the large interval between the consultation and the endoscopy, leading to the prescription of antisecretory drugs, what can be responsible for false results on examinations. Aim - To evaluate changes in ultrarapid urease test and histopathological examination for Helicobacter pylori by antisecretory drugs. Methods - In a prospective double-blind study, 50 patients with dyspeptic complaints and endoscopic diagnosis of peptic ulcer, erosive gastritis, esophagitis or duodenitis, with a positive urease test, were randomized to a 7-day course of treatment with either omeprazole 20 $\mathrm{mg}$ or ranitidine $300 \mathrm{mg}$ a day. Before and after treatment, two biopsy specimens each were obtained from the antrum and corpus and an ultrarapid urease test and a histopathological examination for Helicobacter pylori were performed. Results - There were no significant changes in the results of ultrarapid urease test and histopathological examination for Helicobacter pylori after treatment with ranitidine. With omeprazole, we observed a decrease in positive results in ultrarapid urease test and histopathological examination for Helicobacter pylori in the antrum, but not in the corpus. Conclusion - Omeprazole, used for 7 days, can lead to negative results in ultrarapid urease test and histopathological examination for Helicobacter pylori in the antrum, and should not be employed in patients before the endoscopy is performed.

HEADINGS - Anti-ulcer agents. Helicobacter infections. Omeprazol. Ranitidina. Helicobacter pylori.

\section{REFERÊNCIAS BIBLIOGRÁFICAS}

1. Arvind AS, Cook RS, Tabaqchali S, Farthing MJ. One minute endoscopy room test for Campylobacter pylori [letter]. Lancet 1988;1(8587):704.

2. Atherton JC, Cockayne A, Balsitis M, Kirk GE, Hankey CJ, Spiller RC. Detection of the intragastric sites at which Helicobacter pylori evades treatment with amoxycillin and cimetidine. Gut 1995;36:670-4

3. Bayerdörffer E, Lehn N, Hatz R, Mannes GA, Oertel H, Saverbruch T, Stolte M. Difference in expression of Helicobacter pylori gastritis in antrum and body. Gastroenterology 1992;102:1575-82.

4. Hopkins RJ, Girardi LS, Turney EA. Relationship between Helicobacter pylori eradication and reduced duodenal and gastric ulcer recurrence: a review. Gastroenterology 1996;110:1244-52.

5. Hunt RH. pH and H. pylori - gastric acid secretion and Helicobacter pylori: implications for ulcer healing and eradication of the organism. Am J Gastroenterol 1993;88:481-3.

6. Iwahi T, Satoh H, Nakao M. Lansoprazole, a novel benzimidazole proton pump inhibitor and its related compounds have selective activity against Helicobacter pylori. Antimicrobial Agents Chemother 1991;35:490-6.

7. Lamouliatte H, Cayla R, Daskalopoulos G. Upper digestive tract endoscopy and rapid diagnosis of Helicobacter pylori infection. In: Lee A, Mégraud F, editors. Helicobacter pylori - Techniques for clinical diagnosis \& basic research. London: Saunders; 1996. p.1-16.

8. Logan RPH, Walker MM, Misiewicz JJ, Gummet PA, Karim QN, Baron JH. Changes in the intragastric distribution of Helicobacter pylori during treatment with omeprazole. Gut 1995;36:12-6.

9. Mansi C, Savarino V, Mela GS, Piccioto A, Mele MR, Celle G. Are clinical patterns of dyspepsia a valid guideline for appropriate use of endoscopy? A report on 2253 dyspeptic patients. Am J Gastroenterol 1993;88:1011-5.
10. Marshall BJ, Warren J. Unidentified curved bacilli in the stomach of patients with gastritis and peptic ulceration. Lancet 1984;1:1311-5.

11. Marshall BJ. Campylobacter pylori: its link to gastritis and peptic ulcer disease. Rev Infect Dis 1990;12 Suppl.1:S87-S93.

12. Marshall BJ. Helicobacter pylori. Am J Gastroenterol 1994;89:S116-S128.

13. Maruyama M, Takemoto T, Kondo T. The retrospective study on the accidents associated with the fiberoptic gastroscopy. In: Marcozzi G, Crespi M, editors. Advances in gastrointestinal endoscopy. London: Piccin Medical Books; 1972. p. 203-10.

14. Maton PN. Omeprazole. N Engl J Med 1991;234: 965-75.

15. McNulty CA, Wise R. Rapid diagnosis of Campylobacter pyloridis gastritis. Lancet 1986;1:387.

16. Meining A, Bosseckert H, Caspary WF, Nauert C, Stolte M. $\mathrm{H}_{2}$-receptor antagonists and antacids have an aggravating effect on Helicobacter pylori gastritis in duodenal ulcer patients. Aliment Pharmacol Ther 1997;11:729-34.

17. Mohamed AH, Hunt RH. The rationale of acid suppression in the treatment of acid-related disease. Aliment Pharmacol Ther 1994;8 Suppl.1: 3-10.

18. Pallone F, Luzza F, Delle Fave G, Annibale B, Marcheggiano A, Biancone L, Torsoli A, Capurso L. Lansoprazole and Helicobacter pylori infection. Clin Ther 1993;15 Suppl B:49-57.

19. Price AB. The histological recognition of Helicobacter pylori. In: Lee A, Mégraud F, editors. Helicobacter pylori - Techniques for clinical diagnosis \& basic research. London: Saunders; 1996. p.33-49.

20. Silvis SE, Nebel O, Rogers G, Sugawa C, Mandelstan P. Endoscopic complications. Results of the 1974 American Society for Gastrointestinal Endoscopy Survey. JAMA 1976;235:928-30.

21. Stata Reference Manual. Stata Corporation. Rel 5, 1997. v.1, p.271-307.

22. Stolte M, Bethke B. Elimination of Helicobacter pylori under treatment with omeprazole. Z Gastroenterol 1990;28:271-4. 
Ferreira LEVV de C, Meirelles G de SP, Vieira R La R, Bragagnolo Jr. MA, Chebli JMF, Souza AFM de. Alterações no teste ultra-rápido da urease e no exame anatomopatológico para Helicobacter pylori induzidas por drogas anti-secretoras

23. Stolte M, Meining A, Schmitz JM, Alexandridis T, Seifert E. Changes in Helicobacter pylori induced gastritis in the antrum and corpus during 12 months of treatment with omeprazole and lansoprazole in patients with gastrooesophageal reflux disease. Aliment Pharmacol Ther 1998;12:247-53.

24. Suerbaum S, Leying H, Klemm K. Antibacterial activity of pantoprazole and omeprazole against Helicobacter pylori [letter]. Eur J Clin Microbiol Infect Dis 1991;10:92-3.

25. Tamura K, Murai M, Tanaka M. Activity of lansoprazole (new proton pump inhibitor) against Helicobacter pylori and its therapeutic efficacy [abstract]. Nippon Rinsho 1993;51:3261-6.
26. Thilliainayagam AV, Arvind AS, Cook RS, Harrison IG, Tabaqchali S, Farthing MJ. Diagnostic efficiency of an ultrarapid endoscopy room test for Helicobacter pylori. Gut 1991;32:467-9.

27. Warren J, Marshall B. Unidentified curved bacilii on gastric epithelium in active chronic gastritis. Lancet 1983;1:1273.

28. Weil J, Bell GD, Powell K. Omeprazole and Helicobacter pylori: temporary supression rather than true erradication. Aliment Pharmacol Ther 1991;5:309-13.

Recebido em 11/7/2000. Aprovado em 6/9/2000. 